\title{
Towards the unified non-classical physics: account for quantum fluctuations in equilibrium thermodynamics via the effective temperature
}

\author{
Yu.G.Rudoy, A.D.Sukhanov ${ }^{\dagger}$ \\ People's Friendship University, \\ 117198, 6 Mikluho-Maklaya Str., \\ Moscow, Russia
}

Received May 13, 2005

\begin{abstract}
The concept of effective temperature (ET) $T^{*}\left(T_{0}, T\right)$ is used in order to approximately "quantize" the thermodynamic functions of the dynamical object which is in the thermal equilibrium with thermal bath being at constant temperature $T\left(T_{0}=E_{0} / k_{\mathrm{B}}\right.$, where $E_{0}$ is the ground-state energy, $k_{\mathrm{B}}$ - Boltzmann constant, is the characteristic "quantum" temperature of the system itself). On these grounds the extensive comparative investigation is carried out for the "standard model" of statistical mechanics - the one-dimensional harmonic oscillator ( $\mathrm{HO})$. Three well-known approaches are considered and their thermodynamic consequences thoroughly studied. These are: the exact quantum, or non-classical Planck-Einstein approach, intermediate, or semiclassical Bloch-Wigner approach and, finally, the pure classical, or Maxwell-Boltzmann approach.
\end{abstract}

Key words: equilibrium thermodynamics, effective temperature, quantum fluctuations

PACS: 05.70.-a, 05.30.-d, 05.40.-a

\section{Introduction}

The problems of quantitative and qualitative differences between classical (C) and non-classical (NC) physical "worlds", as well of their interrelations, are ones of the most stringent nowadays (see, e.g., [1-4]). The important role of non-classical ideas in modern physics as well as in natural sciences on the whole was stressed in [5]. Usually one is intended to consider as a non-classical only the QD- "world" of

*E-mail: rudikar@mail.ru

†E-mail: ogol@oldi.ru 
quantum dynamics, but in our opinion it is quite reasonable to include therein the ST- "world" of statistical thermodynamics as well.

The unifying feature of these both "worlds" is the principal need for their probabilistic description and hence for unavoidable use of the concept of fluctuations and their correlations (the quantum and thermal ones, respectively). Obviously, this concept is completely alien to "worlds" of classical dynamics (CD) and classical thermodynamics (CT), which are fully deterministic - at least on the fundamental level. This means that the concepts of statistical ensemble and distribution functions may be sometimes used in classical mechanics as well - e.g., in order to account for the experimental dispersion of the initial conditions for the object's dynamics ${ }^{1}$ (see, e.g. $[7,8])$.

But in principle one can avoid the use of these probabilistic concepts by means of a required exact specification of the initial values. In other words, the classical description is the degenerate case of the non-classical one; namely, all the classical distribution functions may be made to be delta-like (i.e., to have zero spread), whereas in non-classical case this spread is always strictly positive and regulated by the fundamental constants which were introduced in physics by Planck and Boltzmann.

Following this line of thought we adopt (as was already formulated in [14,15]) that there exist in general four large-scale divisions in the description of open physical objects - i.e., interacting with their macroscopic surroundings. These divisions include two levels (upper and lower)), each containing two versions (left and right).

The vertical "level" division includes (from top to bottom) the dynamic, or "cold" D-level and the thermodynamic, or "warm" T-level. It is essential that this division refers not to the physical object itself but rather to its outer surrounding or, more precisely, to the character of the interaction, or the contact between the object and surroundings.

The horizontal "version" division includes (from left to right) classical (C), and non-classical (NC) versions (on the dynamical level the last version is usually called quantum (Q)). In the scope of non-classical versions it is also accepted to consider their restricted variants - the so-called semi- (or quasi-) classical versions (SC or $\mathrm{QC}$ ). The degree of non-classicality in the proposed scheme is determined by the degree of fluctuations taken into account, which are called quantum on the D-level and thermal on the T-level (see for more details in section 2).

It was already noted that on the dynamic level in $\mathrm{C}$-version, the interaction between the object and the surroundings can be described in a fully deterministic (so to say, controllable) way, because it is given unambiguously in terms of macroscopic quantities - external forces or potentials; the results of their effect on the object are also unambiguous (in the case of strictly specified initial conditions).

On the other hand, in Q-version the dynamic effect of the surroundings becomes principally uncontrollable due to the minimal value of action equal to the Planck constant $\hbar$. Thus the description in Q-version is always a probabilistic one, and just

\footnotetext{
${ }^{1}$ One should mention here the rather delicate case of dynamic chaos, which may require a probabilistic (stochastic) description even in the scope of classical Newtonian mechanics due to nonlinearity and ultimate instability caused by small disturbances of initial conditions.
} 
the quantity $\hbar$ is a measure of fluctuations (i.e., dispersion) of conjugate quantum observables. As a mathematical expression of these statements (called sometimes "quantum kinematics") serves the Heisenberg indeterminacies relation, later generalized by Schroedinger.

On the thermodynamic level the outer surroundings, or thermal bath is, as a rule, a macroscopic one, whereas the object may be in general of microscopic origin. The interaction between the object and the bath in this case is called the thermal contact and is also (as in quantum dynamics) an uncontrollable kind of action. In the proposed classification for the T-level we suggest the presence of full thermal equilibrium (or, possibly, slight deviations from it with small fluctuations) between the object and thermostat. This state arises after sufficiently long time of thermal contact, which exceeds the characteristic (for the given object) relaxation time and is characterized by a set of intensive thermodynamic parameters (temperature, pressure, chemical potential and so on).

The thermal contact is of purely statistical origin and consists in random exchanges of some extensive thermodynamic quantities (e.g., inner energy, volume, particle number and so on) between the object and the bath ${ }^{2}$. In the simplest (but seemingly most important) case the thermal equilibrium is characterized by a single intensive thermodynamic variable - the absolute temperature $T$ of the bath. Note that due to zeroth law of thermodynamics the temperature of the object also equals $T$. In classical version of T-level this equality is understood as an exact one, whereas in non-classical - only on the average due to fluctuations.

Summing up, the physical object on the T-level in non-classical version can be described only in the probabilistic terms. Following Einstein, the measure of relevant thermal fluctuations (i.e., dispersions) of thermodynamically conjugate variables (e.g., the inner energy and inverse temperature) is proportional to the Boltzmann constant $^{3} k_{\mathrm{B}}$.

In the classical version on the T-level no such fluctuations are considered, which may be formally viewed as the limit $k_{\mathrm{B}} \rightarrow 0$ (in close analogy with the limit $h \rightarrow 0$ for the version on the D-level).

The most interesting class of physical objects is, in our opinion, that of "warm" microscopic (or "quantum") objects, being in thermal contact with the macroscopic surroundings in the form of thermal bath. In this case both quantum and thermal fluctuations come simultaneously into play: they are due to both types of the uncontrollable effect of the surroundings represented by the fundamental constants $h$ and $k_{\mathrm{B}}$.

Before we formulate the concrete aim of our investigation let us classify more precisely the existing physical theories adopting the above sketched classical vs. non-classical (i.e., "fluctuational") point of view.

\footnotetext{
${ }^{2}$ It is assumed that the thermodynamic state of thermal bath is practically unaffected by this exchange because the degree of macroscopicity of the thermal bath significantly exceeds that of the object.

${ }^{3}$ Einstein [6] attached great importance to this quantity and considered it as a fundamental one - namely, as a "measure of the thermal stability" of the physical object in the thermal bath.
} 


\section{The "fluctuational" classification of physical theories}

On the dynamic, or D-level the relevant fluctuations are the quantum ones of mechanical observables, usually described by the pairs of canonically conjugated variables (e.g., coordinates and momenta). Clearly, purely classic dynamic (CD) theory, which contains no such fluctuations at all, is then the Newton mechanics (including its relativistic generalization by Einstein) for particles as well as Maxwell electrodynamics for fields.

On the other hand, fully non-classical theories of D-level are quantum mechanics and quantum electrodynamics of Heisenberg-Schroedinger-Dirac, because in these theories are inherent the simultaneous fluctuations (as well as correlations) of the both canonically conjugate variables. Now, the Bohr-Sommerfeld mechanics permits the fluctuations of only one variable in each pair and so it is the semiclassical theory of D-level.

Note that the latter theory differs radically from the Newton-Einstein mechanics, because it contains (though not quite consistently) the idea of quantization, and so belongs to the non-classical group of theories of D-level. Clearly, in this group ${ }^{4}$ the relevant fundamental constant is the Planck constant $h$.

Analogously, on the thermodynamic T-level the relevant fluctuations are the thermal ones which apply to thermodynamic observables, which are also combined in pairs of conjugated variables - e.g., the inner energy and (inverse) absolute temperature. On this level one may consider as the classical version the Carno-ClausiusKelvin thermodynamics, which is applicable to all objects being in thermal equilibrium. This theory, like the Newton-Maxwell-Einstein theory on the dynamic D-level, does not contain any idea of fluctuations at all.

On the other hand, a fully non-classical version of thermodynamic (T-level) description is at present the Einstein statistical thermodynamics (which is usually called Einstein's theory of (quasi)thermodynamic fluctuations), because fluctuations of the both of the pair of conjugate thermodynamic parameters (e.g., inner energy and inverse temperature, considered as fully independent quantities) are treated simultaneously on the same footing.

Not surprising that here one also arrives at some kind of Einstein's, or thermodynamic "uncertainties relations". They are formally analogous to the HeisenbergSchroedinger dynamic uncertainties relations, but in some respects differ quite noticeably from the latter (for details see $[7,8]$ and references therein). Clearly, in the group of T-theories the relevant fundamental constant is Boltzmann constant $k_{\mathrm{B}}$.

Finally, as a semiclassical version of the description on the T-level one may consider the approach which was firstly formulated by Szilard [9] and later on was reinterpreted and partly modified by Mandelbrot [10], Tisza [11] and others (see, e.g., the presentation of some of these results in [12]). In the original Szilard's formulation the fluctuations of only one of the pair of conjugated thermodynamic parameters (as a rule, the extensive one - e.g., the inner energy) was considered.

\footnotetext{
${ }^{4}$ For simplicity we consider here only the non-relativistic limit of dynamic description, so the finitness of the velocity of speed $c$ is irrelevant.
} 
Having taken the classification described above, one may well be concerned with a description of the physical object, which is, e.g., classical in dynamic respect and non-classical in the thermodynamic one, or vice versa; some other combinations are also allowed. Evidently, the more fundamental theories are those more fully accounting for fluctuations of both kinds - quantum as well as ther$\mathrm{mal}^{5}$.

Thus the most desirable goal is to establish the logically consistent fully nonclassical description - in both dynamic and thermodynamic senses. Probably, this will appear possible on the grounds of the general theory of open quantum systems (which is now absent) and may, perhaps, deliver the "missing link" even for creating quantum gravity and cosmology ${ }^{6}$.

But for more modest practical purposes some intermediate descriptions are sometimes sufficient, and so various approximate procedures might be of use. The principal possibility of such procedures is based on the fact that the physical object considered as an completely isolated one does not determine unambiguously the choice of its description. More precisely, depending upon the appropriate physical conditions this object may stay in classical or non-classical regimes.

The description in classical regime is often simpler than in non-classical, but the choice between these descriptions is determined not by consideration of simplicity but by numerical values of relevant dimensionless parameters in the condition of the given problem.

As a rule, between these two kinds of descriptions there is some connection; namely, the transition from the non-classical to the classical one is obtained by some kind of approximate semi- (or quasi-) classical procedure, whereas the reverse transition is realized by means of some kind of account (more or less exact) for quantum fluctuations.

On the D-level the parameter of non-classicality is the ratio $h / S_{\mathrm{D}}$, where $S_{\mathrm{D}}$ is the characteristic value of action for the given object in the given physical conditions. If $S_{\mathrm{D}}$ significantly exceeds $h$, the transition from quantum (Q) dynamics to the classical (C) dynamics may be performed by the approximate quasiclassical procedure, or Wentzel-Kramers-Brillouin perturbative expansion in powers of small parameter $h / S_{\mathrm{D}}$.

The reverse transition from C- to Q-regime is also well defined by the quantization rules of Bohr-Sommerfeld (approximate semiclassical description) and HeisenbergSchroedinger-Dirac (exact fully non-classical description).

Analogous classical-non-classical transitions are sometimes possible for the Tlevel of description as well; here other parameters of non-classicality are of use. The most popular of them is the number $N$ of particles in the (macroscopic) physical object. If $N$ is large enough $\left(\sim 10^{23}\right)$, one can perform the transition from the non-

\footnotetext{
${ }^{5}$ From this point of view the "Theory Of Everything" (TOE) actively studied now (see, e.g., [14]) is not more than the elaboration of only one division of the proposed scheme - namely, the non-classical D-theory, because it fully ignores thermal fluctuations.

${ }^{6}$ Note in this connection that the expression for the entropy of the black hole includes all the four fundamental constants entering the Planck's universal system of units - namely $h, c, G$ and especially $k_{\mathrm{B}}$ (see, e.g., [15]).
} 
classical version to the classical one as a perturbative expansion in $1 / \sqrt{N}$, thus neglecting partially or completely the thermal fluctuations.

Clearly, this is not appropriate already for mesoscopic (and all the more for nanoscopic) physical objects with $N \approx 10^{6} \div 10^{2}$, often used nowadays in the experiment at thermodynamic conditions; the neglect of thermal (and, of course, quantum) fluctuations is also quite inappropriate near the critical points as well as at ultralow temperatures [16].

Moreover, the $1 / \sqrt{N}$-expansion does not work at all for such objects as thermal radiation and black holes (where the very concept of $N$ is not defined), so some other (more universal) parameters are needed in these cases. By analogy with the dynamic case, the probable candidate may be the ratio $k_{\mathrm{B}} / S_{\mathrm{T}}$, where $S_{\mathrm{T}}$ is the specific entropy, but this kind of expansion has not yet been sufficiently elaborated.

As was pointed above, the fully non-classical description on the T-level, or Einstein's statistical thermodynamics is formulated as the effective theory (in the sense of [14]) on the grounds of the so-called Boltzmann principle, which is usually considered as one of the statements of the second law of thermodynamics (see [29]). It is important that both descriptions on T-level - Einstein's non-classical, as well as the Carno-Kelvin-Clausius' classical, are applicable to any physical object near its thermal equilibrium with the thermal bath quite independent of the presence or absence of any descriptions for this object on the D-level.

The transition from classical to non-classical version on the T-level is much less investigated compared to the same transition on the D-level. As was pointed above, some ideas of the appropriate stochastization of classical thermodynamics were outlined by Szilard, Mandelbrot and others, see references above), but a consistent and effective theory of this type (not involving any microscopic D-level, or hidden variables theories) is absent so far.

At present, the well developed theory which successfully imitates the semiclassical (in the fluctuational sense!) description on the T-level, is the Maxwell-BoltzmannGibbs' statistical mechanics [13]. This theory is characterized by the account for fluctuatuions of only one (as a rule, the extensive one) thermodynamic quantity, and in order to obtain the fluctuation of the conjugate parameter one should go over to another Gibbs ensemble.

Moreover, the formulation of statistical mechanics (as it follows from the very name of this theory) is founded on the use of the concept of microstates (in the classical or quantum sense - irrelevant in the present aspect) of the physical object. This concept is quite alien to the effective description on the T-level, which should employ only the concept of macrostates.

Nevertheless, there exist a direct and physically appealing way to go over from semiclassical Gibbs approach to a fully non-classical Einstein approach, which is based on the full stochastization of all the pairs of conjugate thermodynamic quantities (see our previous papers $[1,5]$ ).

As was pointed out above, finally we are interested in the full account for both types of fluctuation - thermal as well as quantum ones, but in the present paper we are not intended to go so far as to attempt to build the unified fully non-classical 
description (both on D- and T-levels). We will confine ourselves to a more modest task, which will, however, reveal some general tendencies. Namely, we will show how to build a description which accounts simultaneously for thermal and especially quantum fluctuations, staying within the Gibbs model for the semiclassical version of the T-level description.

The degree of the effect of the object's quantum fluctuations on its thermodynamic behavior at thermal equilibrium depends largely upon the combination of internal and external conditions. These conditions allow the object to stay in C- as well as in Q-regimes during the thermal contact with the thermal bath. It is clear that the conditions of realization of these regimes on the T-level will significantly differ from the corresponding conditions for the D-level (see section 4 below).

Once the non-classical (quantum) version of statistical mechanics based on the Q-regime is achieved, it is also rather simple to obtain its reduced (i.e., semi- or pure classical) versions for C-regime, when one partially or fully neglects the quantum fluctuations. This $\mathrm{Q} \rightarrow \mathrm{C}$ transition is achieved by means of high-temperature expansion in powers of $1 / T$, always accompanied by simultaneous quasiclassical expansion in powers of $h$.

These both expansions are, of course, only formal ones; as a matter of fact, the expansion (like the situation in dynamical case) goes in powers of dimensionless parameter - in this case the ratio $h \nu / k_{\mathrm{B}} T$, where $\nu$ is some characteristic frequency, which may be always assigned to the object, $T$ is the absolute temperature of the thermostat.

Much less trivial task, with which we will be mostly engaged here, is the reverse $\mathrm{C} \rightarrow \mathrm{Q}$ transition, which demands including in some way (as a rule, only approximately) the quantum fluctuations (i.e., the existence of Q-regime), starting from the classic dynamic description (i.e., from the C-regime) of the given object.

The principal way of performing such a transition was originally sketched in the works by E. Wigner and F. Bloch early in the 30-ies of the 20th century, presented by them in the remarkable and up till now often cited papers ${ }^{7}$ [22] and [23]. These authors have simultaneously and independently developed rather different theoretical approaches in the field of quantum statistical mechanics; in particular, in the articles cited above such fruitful concepts as Wigner function and Bloch equation were introduced. Loosely speaking, these authors for the first time managed (though in somewhat different ways) to account for quantum (i.e., dynamical fluctuational) corrections in the thermodynamic description of the given physical object .

Wigner [22] has built the quasi-classical statistical mechanics for the object in the arbitrary external field, using the expansion in powers of the small quantity $h$ and (simultaneously) in powers of the large quantity $T$. In fact, as was pointed out, this expansion is in powers of really small dimensionless ratio $h \nu / k_{\mathrm{B}} T$, where $\nu$ is some characteristic frequency for the object. Wigner found only some first terms of this expansion (which complexity rises very fast with the number of terms) for the partition function as well as for distribution functions in coordinate $q$ and momentum $p$.

\footnotetext{
${ }^{7}$ Slightly modified exposition of these results may be found in modern textbooks on statistical mechanics [18-21]; see also the footnote 8 below.
} 
Unlike Wigner, Bloch used more general approach, which does not employ any perturbative ideology (i.e., the expansion in some small parameter). Bloch [23] got the exact (i.e., valid for all temperatures) expressions for the distribution functions (d.f.'s) independently in $q$ and $p$ only for the particular case of one-dimensional harmonic oscillator (1-d HO). Thus Bloch fully took into account the existence of Q-regime; it is not surprising that in the limit of C-regime the Bloch's result coincides with the Wigner's one for the particular case ${ }^{8}$ of 1-d HO.

Nevertheless, exact Bloch's (or, equivalently, refined Wigner's) result remains to be only approximate for the partition function (p.f.) in Q-regime because the very connection between d.f.'s and p.f. in this case is rather nontrivial (more details may be found in section 8). These results bring about a significant improvement over the ordinary classical version (accounting for the existence of C-regime only), especially in the region of low temperatures, where the quantity $h \nu / k_{\mathrm{B}} T$ cannot be considered as a small one. But as will be shown below, in some respects these results differ significantly from the exact results for the p.f. of the 1-d HO, obtained by Planck and Einstein.

Our main aim in the present paper is as follows. We are going to show that most of the results by Wigner and Bloch may be obtained in a unified - and, which is even more important - much simpler way using the notion of the generalized, or effective temperature (ET). We shall consider in detail only the case of 1-d HO, which possess an exact solution obtained by Planck and Einstein and so permits the control of accuracy for various approximations.

But it seems to us that the way suggested here possesses much wider (if not universal) range of applicability if one takes into account the universality of the concept of ET itself (section 4) and especially its connection with the third law of thermodynamics (section 5) in its present formulation.

The main advantage of our approach consists in avoiding any dynamic aspects connected with the transition from Q- to C-regimes (e.g., the intricate enough WKBtheory). All you need to know about the corrections into thermodynamic quantities due to quantum fluctuations seems to be already incorporated in the ET; the preliminary version of this approach was suggested in [26] (for a fuller discussion see the next section 4)).

Seemingly, this circumstance reflects partly the universality (in the sense of specific model independence) of the general thermodynamic laws contained already in classical version of the T-level, which do not distinguish between the Q- or C-regimes altogether. These regimes differ only in the specific forms of the dependence of thermodynamic quantities upon the ratio $h \nu / k_{\mathrm{B}} T$; just this difference is partly taken into account by the ET. As is the case for any effective (in the sense of [14]) theory, the relevant quantities are not only calculated but also experimentally measured according to their operational definitions.

\footnotetext{
${ }^{8}$ About half a century later it was revealed $[24,25]$ that after the appropriate (mathematically sufficiently hard) refining of Wigner's approach it becomes valid for arbitrary (and not only small) values of $h \nu / k_{\mathrm{B}} T$. This refined Wigner's approach when applied to the 1-d HO leads to the same exact results for d. f.'s as the Bloch's one.
} 
Contrary to the $\mathrm{C}$-version of thermodynamics, the statistical mechanics is always based on some version (C- or Q-) of dynamics and so calculated thermodynamic functions are more or less dependent upon the model of the object. So by refining the statistical-mechanical description (i.e., going from C- to SC- or even Q-version for the macroscopic object) one should operate with many dynamic variables in the high-dimensional space ${ }^{9}$ - phase space (for C-regime) or Hilbert space (for Qregime) and then to average upon these variables.

In this respect the method of ET provides a great economy, because it allows for any objects during the $\mathrm{C} \rightarrow \mathrm{Q}$ transition to deal instead only with few smoothbehaving thermodynamic quantities in the low-dimensional space of thermodynamic variables. Of course, the simplification of the mathematical apparatus permits to better control the physical sense of the obtained results. Moreover, the results of thus improved descriptions should be in better accordance with the experimental thermodynamic measurements ${ }^{10}$.

To avoid confusion we should note in conclusion of this section that despite its effectiveness, the method of ET (which is described in sections 4, 5) presents only some kind of extrapolation (from C- to Q-regime in the scope of semiclassical description on the T-level) and so from the very beginning is an approximate one.

In sections 6-9 we shall give the evaluation of applicability and accuracy of the ET-method and thus establish more distinct boundaries between the classical and quantum versions of Gibbs equilibrium statistical mechanics. The latter theory is briefly sketched in section 3; the description of HO in C-regime (Maxwell-Boltzmann approach) is given in section 6 and in Q-regime (Planck-Einstein approach) - in section 7. Sections 8 and 9 are devoted to the semiclassical Bloch and Wigner approaches, whereas in section 10 some conclusions are formulated.

\section{Gibbs statistical mechanics}

To describe the physical object on the grounds of Gibbs statistical mechanics (or SCT-theory according to the nomenclature suggested in section 2) means to calculate the appropriate thermodynamic functions in terms of the most fundamental quantity of Gibbs theory - the partition function (p. f.) $Z(T)$. The starting expressions for $Z(T)$ differ for Q- and C-regimes, whereas the recipes (but of course not the results) for further calculation of thermodynamic functions in both cases are quite identical.

Namely, if the p.f. $Z(T)$ is known for the object, so the Massieu-Planck function $\Phi(T)$ and free energy $F(T)$ are of the form

$$
\Phi(T)=\ln Z(T), \quad F(T)=-k_{\mathrm{B}} T \Phi(T) .
$$

Inner energy $E(T)$ and specific heat capacity $C_{\mathrm{V}}(T)$ at constant volume $V$ have

\footnotetext{
${ }^{9}$ One should note that these variables may - and, as a rule, really possess - rather complex and irregular behavior.

${ }^{10}$ Note that just this way led Planck [27], who studied as the physical object the thermal radiation, finally to the invention of his famous "quantum" of action $h$.
} 
correspondingly the forms

$$
\begin{aligned}
E(T) & =k_{\mathrm{B}} T^{2}[\mathrm{~d} \Phi(T) / \mathrm{d} T]=k_{\mathrm{B}} T^{2}[(1 / Z(T))(\mathrm{d} Z(T) / \mathrm{d} T)] \\
C_{\mathrm{V}}(T) & =\mathrm{d} E(T) / \mathrm{d} T=2(E(T) / T)+k_{\mathrm{B}} T^{2}\left[\mathrm{~d}^{2} \Phi(T) / \mathrm{d} T^{2}\right]
\end{aligned}
$$

and, finally, the entropy $S(T)$ is given by the relation

$$
S(T)=-\mathrm{d} F(T) / \mathrm{d} T=E(T) / T+k_{\mathrm{B}} \Phi(T) .
$$

Any given physical object on the dynamic level is described by the Hamilton function $H(q, p)$ (in $C$-regime) or by Hamiltonian $\hat{H}(q, p)$ (in $Q$-regime); in the simplest case of one particle in the potential $U(q)$ one obtains:

$$
H(q, p)=\left(p^{2} / 2\right)+U(q), \quad \hat{H}(q, p)=-\left(h^{2} / 2\right)\left(\mathrm{d}^{2} / \mathrm{d} q^{2}\right)+U(q) .
$$

Here $H(q, p)$ is a function, determined uniquely on the phase $\operatorname{space}^{11}(q, p)$ of coordinates $q$ and momenta $p$, while $H(q, p)$ is an operator with eigenvalues $E_{n} \geqslant 0$ ( $n=0,1,2, \ldots-$ main quantum number). In terms of these quantities the p.f. $Z(T)$ is given in the following ways: for $C$-regime

$$
Z^{\mathrm{C}}(T)=\int \mathrm{d} q \mathrm{~d} p(2 \pi \hbar)^{-s} \exp \left[-H(q, p) / k_{\mathrm{B}} T\right]=\int \mathrm{d} E g(E) \exp \left[-E / k_{\mathrm{B}} T\right],
$$

where $g(E)$ is the phase space structure function, or density of states ( $s$ is the number of degrees of freedom), whereas for Q-regime

$$
Z^{\mathrm{Q}}(T)=\sum_{n} g_{n} \exp \left[-E_{n} / k_{\mathrm{B}} T\right]
$$

where $g_{n}$ is the degeneration factor for $n$-th energy level $E_{n}$. Strictly speaking, the expression (6) cannot be considered as a pure classic one, because the ultimate quantum origin of the object is partially taken into account (though in a rather primitive way) by using the factor $(2 \pi \hbar)^{-s}$. Following $P$. and $T$. Ehrenfest, one introduces the notion of "cells" in phase space, which partially reflects the existance of uncertainties relation between $q$ and $p$. Obviously, the "cellular" structure of the phase space is not more than some kind of quantum "kinematics", whereas the quantum dynamics should be (and in fact will be) accounted for more adequately below. Note that due to the notion of "cells" the Planck constant enters the "classical" expression $Z^{\mathrm{C}}(T)$ from the very beginning in order to obtain it as the limit of $Z^{\mathrm{Q}}(T)$ when (formally) $h \rightarrow 0$. Probably, it would be more correct to substitute the differential element $\mathrm{d} p \mathrm{~d} q$ of phase space volume in (6) by the product of finite differences $\Delta p \Delta q$, so that the relation $\Delta p \Delta q / h^{s}$ would be an integer number (usually very large). Then the notation employed in (6) corresponds to the "small $h$ " limit, because only in

\footnotetext{
${ }^{11}$ Here and below we use the renormalized coordinates $q \rightarrow q \sqrt{m}$ and momenta $p \rightarrow p / \sqrt{m}$ ( $m$ is the mass of the particle), so that this scale transformation is a canonical one and the phase space volume element $\mathrm{d} p \mathrm{~d} q$ remains invariant.
} 
this case $\Delta p \Delta q / h^{s} \rightarrow \mathrm{d} p \mathrm{~d} q$ as $h \rightarrow 0$. The procedure of "cell quantization" represents the different ways of determining and counting the number of microstates of the given object. In C-regime these states form a strictly continuous set, and so their number is always infinite. In Q-regime the states may form a discontinuous, or discrete set, denoted usually by some (also discrete) quantum numbers. Clearly, there may well exist the continuous part of the state (not phase!) space, so the full number of states may be also infinite (though in many cases countable).

But the most important thing is the following: in Q-regime the uncertainties relations prevent the description of the phase space states by means of simultaneous exact specification of both $p$ and $q$, whereas in C-regime it is quite legitimate. Obviously, in the intermediate, or SC-regime the demands of the quantum uncertainty principle (and appropriate relations) should be taken into account more carefully (as compared to simple "cell quantization").

We propose (section 4) some sort of this procedure, which (in contrast to Bloch and Wigner) is performed by means of the ET after the Gibbs averaging over the phase space is fulfilled. Of course, it is a rather difficult task to check the accuracy of such a procedure for obtaining $Z^{\mathrm{SC}}(T)$ in the general case, when the exact solution for $Z^{\mathrm{Q}}(T)$ is absent ${ }^{12}$.

Evidently, the integration upon the continuous variables $(q, p)$ for obtaining $Z^{\mathrm{C}}(T)$ is, in general, much simpler than the summation upon the discrete energy eigenvalues $E_{n}$ necessary for obtaining $Z^{\mathrm{Q}}(T)$ (separating the problem of finding these $E_{n}$ even for known $\left.\hat{H}(q, p)\right)$. Clearly, both mathematical procedures (for Cand Q-regimes respectively) are in general not equivalent to each other. Indeed, they lead via $\mathrm{Z}(\mathrm{T})$ from (6) and (7) to thermodynamic functions (1)-(4) with quite different dependence upon $T$; as a rule, this dependence approximately coincides at high $T$, but differs radically at low $T$ (see in this connection any advanced textbook, e.g., $[12-15])$.

More precisely, the choice among the expressions (6) and (7) - or, which is the same, among the type of dynamic, or microscopic regime (C- or $\mathrm{Q}-$ ) the object remains in thermal equilibrium - is regulated by the dimensionless temperature parameter

$$
c=E_{0} / k_{\mathrm{B}} T=T_{0} / T, \quad \text { where } \quad T_{0} \equiv E_{0} / k_{\mathrm{B}},
$$

which may be called the degree of object's "thermal quantumness".

Here $E_{0}$ is the lowest possible, or ground-state energy of the object, $T_{0}$ - the corresponding quantum dynamic characteristic quantity, having the dimension of temperature; the quantity $k_{\mathrm{B}} T$ has the meaning of some "average" thermal energy, which approximately relates to one degree of freedom for thermostat (considered as the classical one).

Obviously, the almost pure C-regime corresponds to the low values of $c$, when $T$ is much higher than $T_{0}\left(T \gg T_{0}\right.$, or in the limiting case $\left.T \rightarrow \infty, c \rightarrow 0\right)$, whereas the almost pure Q-regime corresponds to the high values of $c$, when $T$ is much lower

\footnotetext{
${ }^{12}$ That is why we employ in this paper as an example just the one-dimensional HO - the simple enough, but by no means trivial model, fortunately possessing the exact solution $Z^{\mathrm{Q}}(T)$.
} 
than $T_{0}\left(T \ll T_{0}\right.$, or in the limiting case $\left.T \rightarrow 0, c \rightarrow \infty\right)$; note, that for $T_{0}=0$ (purely classical object) the C-regime is formally valid for all $T>0$.

In other words, to be in the C-regime for a thermodynamic system on the whole means that the dynamic object should be in thermal contact with a "warm enough" thermostat, whereas to be in a Q-regime - on the contrary, with a "cold enough" thermostat.

Consequently, the description of the quantum object in the thermostat depends not only upon the temperature of the thermostat (as it usually holds for the classical object), but also upon the property of the quantum object itself (namely, its characteristic temperature $\left.T_{0}\right)$. Therefore it is not surprising that both p.f.'s $Z^{\mathrm{C}}(T)$ and $Z^{\mathrm{Q}}(T)$ are in fact the functions of $c$, while the $Z^{\mathrm{C}}(T)$ is the high- $T$ (low-c) limit of its quantum counterpart $Z^{\mathrm{Q}}(T)$.

Here naturally does arise the opposite question: if there is given $Z^{\mathrm{C}}(T)$, is it possible to propose some procedure which could give us some knowledge about the behavior of its quantum counterpart $Z^{\mathrm{Q}}(T)$ ? Of course, the question is to describe not only the high- $T$ (low- $c$ ) but also the most interesting low- $T$ (or high- $c$ ) limit.

Although the existence of such a procedure is in no way a priori obvious, there are quite convincing arguments in its favor. These arguments follow unambiguously from the results of Bloch and Wigner cited and briefly discussed in section 2 (for a fuller discussion see sections 8 and 9, respectively).

In the context of our paper the most significant thing is that these authors in fact discovered a tool for carrying out the approximate semiclassical (SC) procedure that we are interested in. This tool in its very essence proves to be nothing else than the meant above effective temperature (ET) (see section 4-5); at first glance the approaches by Wigner and Bloch seem to have little to do with this concept ${ }^{13}$, but in section 7-9 we show the opposite.

Being an approximate one, the semiclassical procedure in the case of Wigner (section 9) supplies us with some additional knowledge only of high- $T$ behavior for $Z^{\mathrm{Q}}(T)$ (though for arbitrary $U(q)$ ), whereas in the case of Bloch (section 8) one also obtains some knowledge about the much more intriguing low- $T$ region for this quantity (but only for the HO case).

\section{The general concept of effective temperature}

The concept of ET (which will be here and below denoted by $T^{*}$ ) is rather universal and applicable to any physical object (which are all ultimately of quantum origin). The object here and below is assumed to be in thermal equilibrium with the thermostat, possessing the absolute temperature $T \geqslant 0$.

In general $T^{*} \neq T$ (or, more definitely, it appears that always $T^{*} \geqslant T$ ), because the ET depends not only upon the "outer" ordinary, or "classical" temperature $T$,

\footnotetext{
${ }^{13}$ This notion was explicitly introduced (but not discussed) much later by Landau and Lifshitz [8] only in connection with Wigner's result [22]. Unfortunately, there was not established any connection between the reproduced in [18] results by Wigner and Bloch, as well as their thermodynamic consequences were not analyzed.
} 
but also upon "inner" characteristic, or "quantum" temperature $T_{0}$ defined by (8), so as

$$
T^{*}=T^{*}\left(T_{0}, T\right), \quad T^{*} \geqslant T, \quad T_{0} \geqslant 0 .
$$

Obviously, one can say that the (positive) difference between $T^{*}$ and $T$ is fully determined by the inherently quantum nature of the object.

As is well known, in general $E_{0}$ is non-zero (positive) quantity $\left(E_{0}>0\right)$, and so is $T_{0}>0$; this is due to the fluctuations of purely quantum origin. But the precise value of $E_{0}$ significantly depends upon the physical nature of the relevant object's degree of freedom.

For example, for the translational motion of the quantum object in the macroscopic (large enough) box one has $E_{0} \approx 0$, so that $T_{0} \approx 0$ and for all $T$ holds:

$$
T^{*}(0, T) \approx T \text {. }
$$

But for most internal degrees of freedom of the single quantum object one has $E_{0}>0, T_{0}>0$, so in the low-temperature limit $T \rightarrow 0$ it holds

$$
T^{*}\left(T_{0}, 0\right)=T_{0} .
$$

Thus, ET should interpolate between the "external" temperature T of the thermostat and the "internal" temperature T0 of the object itself. Generally speaking, one may expect, that for different classes of physical objects the functional form of (9) is also different, although the very concept of ET seems to be a universal one.

Further, on rather general grounds caused by the very structure of the expressions (6) and (7) (see also section 7) all the thermodynamic functions (1)-(4) do depend not upon $T_{0}$ and $T$ apart, but upon their ratio $c$. So one should also anticipate the self-similar, or "scaling" character of the ET dependence (9) upon $T_{0}$ and $T$, namely,

$$
T^{*}\left(T_{0}, T\right)=T^{*}(c), \quad c=T_{0} / T .
$$

This is much more physical, because the high- and low-T limits may be achieved now not only in formal limits for the temperature itself $(T \rightarrow \infty$ and $T \rightarrow 0)$, but in essentially less stringent ones for the dimensionless temperature parameter $(c \rightarrow 0$ means only $T \gg T_{0}$ and $c \rightarrow \infty-$ accordingly $T \ll T_{0}$ ).

Loosely speaking, in the first limit the system almost fully "loses" its quantum features (though $T$ may be finite, but high enough to largely exceed $T_{0}$ )

$$
T^{*}(c) \rightarrow T_{C^{*}} \equiv T(c \rightarrow 0) .
$$

In the opposite limit the system, on the contrary, becomes almost fully "quantum degenerate" (though $T$ may strongly differ from zero, but at the same time being much less than $T_{0}$ ), so that

$$
T^{*}(c) \rightarrow T_{Q}^{*} \equiv T_{0}(c \rightarrow \infty) .
$$

For the future purposes it is convenient to introduce besides c from (8) two additional dimensionless parameters, or relative effective temperatures, namely

$$
T^{*}(c) / T=f(c), \quad T^{*}(c) / T_{0}=\varphi(c), \quad \varphi(c)=f(c) / c,
$$


so that for all values of $c$

$$
T^{*}(c)=T f(c)=T_{0} \varphi(c) ; \quad f(c), \varphi(c) \geqslant 1 .
$$

It is instructive to calculate also the derivatives of ET on $T$. Denoting here and below the derivative on the variable $c$ by prime, we have

$$
f^{\prime}(c)=\varphi(c)-c \psi(c), \quad \psi(c) \equiv \varphi^{2}(c)-1 \geqslant 0
$$

while according to the definition (8)

$$
\mathrm{d} c(T) / \mathrm{d} T=-c / T=-c^{2} / T_{0} \leqslant 0, \quad T \mathrm{~d} c / \mathrm{d} T=-c \leqslant 0 ;
$$

finally one obtains:

$$
\mathrm{d} T^{*}(c) / \mathrm{d} T=f(c)+f^{\prime}(c) T[\mathrm{~d} c(T) / \mathrm{d} T]=c^{2} \psi(c) \geqslant 0
$$

Further, noting that $\psi^{\prime}(c)=-2 \varphi(c) \psi(c)<0$, one easily finds:

$$
\begin{aligned}
& \mathrm{d}^{2} T^{*}(c) / \mathrm{d} T^{2}=\left(2 / T_{0}\right) c^{3} \psi(c)[f(c)-1] \\
& \mathrm{d}^{3} T^{*}(c) / \mathrm{d} T^{3}=\left(2 / T_{0}^{2}\right) c^{4} \psi(c)\left[3(f(c)-1)^{2}-c^{2}\right] .
\end{aligned}
$$

Note, that both equations (19) and (20) are strictly positive for all values of $c$. However the latter equation $(21)$ is alternating in sign and has a zero at $c_{0}=$ $\sqrt{3}\left[f\left(c_{0}\right)-1\right]$; so the equation $(20)$ possesses maximum at $c_{0}$, whereas the equation $(21)$ - the inflection point. Let us remark in conclusion that in order to make not only qualitative but also quantitative predictions one should, of course, obtain some explicit expression for ET, which is defined in general form in (16).

\section{Effective temperature and the third law of thermodynamics}

The physical meaning of the term "effective temperature" (ET) and its connection with the third law of thermodynamics can be seen most natural when one applies ET to the concept of object's inner energy $E(T)$. To start with, we recall one of the most prominent results of the classical statistical mechanics (see, e.g., [12-15]) namely, the virial theorem ${ }^{14}$ (or, as it is sometimes called, "equipartition" of the inner energy in the degrees of freedom):

$$
E^{\mathrm{C}}(T)=s \alpha k_{\mathrm{B}} T, \quad C_{\mathrm{V}}^{\mathrm{C}}=s \alpha k_{\mathrm{B}}=\text { const. }
$$

The first of these expressions states the presence of equal portions $\alpha k_{\mathrm{B}} T$ of the inner energy per each of the s object's degrees of freedom; here $\alpha=1 / 2+1 / l$, where $l$ is the homogeneity index of the potential energy function $U(q)$; in particular, for the

\footnotetext{
${ }^{14}$ This theorem may be easily obtained from the Maxwell-Boltzmann classical version of Gibbs approach (see section 6) on the grounds of classical p.f. (6) and the definitions (2) and (3).
} 
HO $l=2$, so $\alpha=1$ (later on we consider only the case $s=1$ ). So for one-dimensional CHO one obtains

$$
E^{\mathrm{C}}(c)=E_{0}(1 / c), \quad C_{\mathrm{V}}^{\mathrm{C}}=k_{\mathrm{B}} .
$$

The second expressions in (22) and (23) follow immediately from the first ones and confirm the independence of specific heat upon the temperature $T$.

The latter statement is, unfortunately, in an obvious contradiction with the third law of thermodynamics, or Nernst theorem, which maintains:

$$
C_{v}(T) \rightarrow 0 \quad(\text { as } T \rightarrow 0 \text { or } c \rightarrow \infty) .
$$

This contradiction shows that the "equipartition" theorem is significantly violated at low $T$-region (the experiment also confirms this fact).

The resolution of this difficulty on the phenomenological level is well known: one usually hears about the "effective" (i.e., depending upon the thermostat temperature $T)$ number $s(T)$ of object's degrees of freedom. It is assumed that in the low- $T$ (high$c)$ region they are "frozen out", so that $s(T)>0$ at $T>0$, and so does the specific heat $C_{v}(T)$.

Physically such an explanation is qualitatively quite acceptable, because it eliminates the contradiction with the third law of thermodynamics, which is inherent to $C_{\mathrm{V}}^{\mathrm{C}}$ from the equations (22) or (23). Indeed, this simply means that at low enough values of $T$ the system's degrees of freedom are almost not activated. This in turn signifies (as is most clearly demonstrated by (24)), that the system cannot effectively exchange the energy in the form of heat with the thermostat.

But it seems much more natural and convincing to let the number $s$ of object's degrees of freedom be constant (as it really is) and introduce instead of $s(T)$ into the first expressions in equations (22) and (23) for the inner energy in place of $T$ the ET defined in (15). Then one obtains ${ }^{15}$

$$
E^{\mathrm{Q}}(T)=E^{\mathrm{C}}\left[T^{*}(c)\right]=E^{\mathrm{C}}(T) f(c)
$$

and, taking into account equation (4), also

$$
C_{\mathrm{V}}^{\mathrm{Q}}(T)=\mathrm{d} E^{\mathrm{Q}}(T) / \mathrm{d} T=C_{\mathrm{V}}^{\mathrm{C}}\left[\mathrm{d} T^{*}(c) / \mathrm{d} T\right]=C_{\mathrm{V}}^{\mathrm{C}} c^{2} \psi(c) .
$$

Equation (25) obviously violates the "theorem" of equipartition (which is of course valid only classically), because the ET, defined by equation (15), depends not only upon $T$ but also upon $T_{0}$ and thus is different for every degree of freedom, which may be of various physical nature and possesses different values of $T_{0}$.

The equation (26) should fulfill the condition (24) and, consequently, the function $\psi(c)$ should go to zero by $c \rightarrow \infty$ not slowly than $1 / c^{3}$. Note once more, that the demand (24) of Nernst theorem signifies in fact that the heat exchange between the quantum system and the thermostat is strongly suppressed at low $T$.

By the latter argument not only the third law of thermodynamics but also the zeroth law (in slightly modified form) may be fully explained. At high enough values

\footnotetext{
${ }^{15}$ Note that the equations (25) and (26) are the exact ones in the case of QHO for the particular choice of $f(c)$ (see section 7 ), and so we take them as heuristically valid also in the general case.
} 
of $T$ (low $c$ ) any system behaves as almost classical one (as if $E_{0} \equiv 0$ ), and so due to equation (13) $T^{*} \equiv T$. As the thermostat temperature $T$ lowers (accordingly, $c$ rises) the value of $T^{*}$ differs from $T$ more and more.

Clearly, owing to Nernst theorem (24) the object becomes less and less capable of exchanging heat with the thermostat. In other words, in the limit $T \rightarrow 0(c \rightarrow \infty)$ the object in fact ceases to be in thermal contact with the thermostat and gradually goes over to the adiabatically isolated system. So it is not surprising that $T^{*}$ becomes finally quite independent of $T$ and equals $T_{0}$. Note that for almost "classical" object (i.e., object with $T_{0} \approx 0$ ) the approximate equality $T^{*} \approx T$ holds practically in the whole interval of $T$.

\section{Maxwell-Boltzmann classical approach}

One of the most important notions in statistical mechanics is the notion of distribution function (d.f.) - as a rule, in coordinates $q$ and momenta $p$ (sometimes also in energy $E$ and, maybe, other dynamic variables). For the separable Hamiltonian of the form (5) these d.f.s for the system in $C$-regime are the familiar one Maxwell d.f.

$$
P^{\mathrm{C}}(p ; T)=\left[Z_{p}^{\mathrm{C}}(T)\right]^{-1} \exp \left[-\left(1 / k_{\mathrm{B}} T\right)\left(p^{2} / 2\right)\right]
$$

and Boltzmann d.f.

$$
P^{\mathrm{C}}(q ; T)=\left[Z_{q}^{\mathrm{C}}(T)\right]^{-1} \exp \left[-\left(1 / k_{\mathrm{B}} T\right) U(q)\right]
$$

Of course, for the object in C-regime it is quite legitimate to speak about the simultaneous or joint d.f. in the system's phase space, i.e. about the Gibbs d.f.

$$
P^{\mathrm{C}}(p, q ; T)=P^{\mathrm{C}}(p ; T) P^{\mathrm{C}}(q ; T)=\left[Z^{\mathrm{C}}(T)\right]^{-1} \exp \left[-\left(1 / k_{\mathrm{B}} T\right) H(p, q)\right] .
$$

Due to statistical independence of the distribution in $p$ and $q$ in this case (or, which is just the same, the absence of any correlations among $p$ and $q$ ) the joint d.f. (29) is given by the product of d.f.s (27) and (28). In other words, the d.f. (29) possesses a physical meaning since from the classical point of view every phase space point $(p, q)$ is always exactly defined.

The normalization factor in (29) is obviously equal to the product of the corresponding factors in (27) and (28),

$$
Z^{\mathrm{C}}(T)=Z_{p}^{\mathrm{C}}(T) Z_{q}^{\mathrm{C}}(T)
$$

and thus coincides with the expression (6) for the p.f. of any object (described in terms of $H(p, q))$ in C-regime.

For the particular case of $\mathrm{HO}$ (see footnote 13) one has

$$
U(q)=(1 / 2) \omega^{2} q^{2},
$$

so both d.f.s (27) and (28) get the Gaussian form

$$
\begin{aligned}
P^{\mathrm{C}}(p ; T) & =\left[Z_{p}^{\mathrm{C}}(T)\right]^{-1} \exp \left[-\alpha_{p}(T) p^{2}\right] \\
P^{\mathrm{C}}(q ; T) & =\left[Z_{q}^{\mathrm{C}}(T)\right]^{-1} \exp \left[-\alpha_{q}(T) q^{2}\right]
\end{aligned}
$$


Using twice the Poisson integrals, one obtains the partial p.f.s in the form

$$
Z_{p, q}^{\mathrm{C}}(T)=(2 \pi \hbar)^{-1 / 2}\left[\pi / \alpha_{p, q}(T)\right]^{1 / 2}
$$

where

$$
\alpha_{p}(T)=1 / 2 k_{\mathrm{B}} T, \quad \alpha_{q}(T)=\omega^{2} \alpha_{p}(T)=\omega^{2} / 2 k_{\mathrm{B}} T .
$$

Then it follows from (30), (34) and (35) that

$$
Z^{\mathrm{C}}(T)=k_{\mathrm{B}} T / \hbar \omega, \quad \text { or } \quad Z^{\mathrm{C}}(c)=1 / 2 c
$$

and hence, according to (2)-(4), the equation (23) as well as the expression for the entropy

$$
\left(1 / k_{\mathrm{B}}\right) S^{\mathrm{C}}(c)=\sigma_{0}-\ln c, \quad \sigma_{0}=1-\ln 2, \quad 0<\sigma_{0}<1,
$$

are easily obtained. Evidently, $S^{\mathrm{C}}(c) \rightarrow-\infty$, i.e. diverges logarithmically as $c \rightarrow \infty$, and so the quantity $S^{\mathrm{C}}(c)$ (as well as $C_{\mathrm{V}}^{\mathrm{C}}$ ) is in strong contradiction with another demand of the Nernst theorem: the value of $S(T)$ as $T \rightarrow 0$ should be a finite nonnegative one (presumably zero).

\section{Planck-Einstein non-classical approach}

As mentioned above, the use of $\mathrm{HO}$ as a test model is justified, because the study of this model in various approximations (both theoretically and experimentally) has a rather long history and is well familiar. Moreover, due to Planck [27] and Einstein [29] we possess the exact solution $Z^{\mathrm{Q}}(T)$ for the one-dimensional QHO with $s=1$.

Perhaps, Wien was the first who proved the self-similar, or "scaling" form of spectral energy d.f. (and hence of all thermodynamic functions) in the theory of thermal radiation ${ }^{16}$. According to Wien [27], the expression (8) for $c$ should be of the form $c=\alpha(v / T)$ ( $\alpha=$ const), and it was Planck [27] who pretty soon showed that $\alpha=h / k_{\mathrm{B}}$.

Further on, using mainly the thermodynamic arguments Planck [27] deduced an explicit functional form for the Kirchhoff-Wien's spectral energy d.f. and the inner energy of HO, whereas Einstein [29] calculated the p.f. for HO. In fact, Planck for the first time "quantized" the energy of HO, but only later he managed [28] to account for the zero-point energy $E_{0}=1 / 2 \cdot \hbar \omega$, where $\omega$ is the characteristic frequency of $\mathrm{HO}$. Obviously, for QHO the characteristic "quantum" temperature is of the form

$$
T_{0}=E_{0} / k_{\mathrm{B}}=\hbar \omega / 2 k_{\mathrm{B}} \quad(\mathrm{QHO}),
$$

where $\omega$ is the characteristic frequency of $\mathrm{HO}$.

It follows from $[27,29]$ that according to the definitions (16) and (25) for $T^{*}(c)$ it is natural to define the Planck-Einstein ET by the following functions:

$$
\varphi(c)=\operatorname{cth} c, \quad f(c)=c \operatorname{cth} c,
$$

\footnotetext{
${ }^{16}$ Long before Kirchhoff [31] stressed that this function should be a universal one, i.e. should not depend upon the physical nature of particular radiating body or thermal bath. Note that both Kirchhoff and Wien used pure thermodynamic arguments following the traditions of Carno.
} 
with the well known asymptotic expansions

$$
\varphi(c)= \begin{cases}(1 / c)\left[1+(1 / 3) c^{2}-(1 / 45) c^{3}+\cdots\right], & (c \rightarrow 0), \\ 1+2 e^{-2 c}\left(1+e^{-2 c}+\cdots\right), & (c \rightarrow \infty)\end{cases}
$$

and, accordingly,

$$
f(c)= \begin{cases}1+(1 / 3) c^{2}+O\left(c^{3}\right), & (c \rightarrow 0), \\ c\left[1+O\left(e^{-2 c}\right)\right], & (c \rightarrow \infty) .\end{cases}
$$

Both expansions (40) and (41) obviously agree with the expected behavior of ET (13) and (14). Using definition (17) and expansion (40), we further have

$$
\psi(c)= \begin{cases}(1 / c)^{2}\left[1-(1 / 3) c^{2}+O\left(c^{4}\right)\right], & (c \rightarrow 0), \\ 4 e^{-2 c}+O\left(e^{-4 c}\right), & (c \rightarrow \infty) .\end{cases}
$$

By means of equations (40)-(42) it is not difficult to determine the low- and high-c asymptotes of all the ET derivatives (19)-(21); in particular, the first derivative (19) shows the behavior, which completely satisfies the demands of classic theory at low $c$ and Nernst theorem at high $c$ for the specific heat (26).

The exact p.f. ZQ(c) for the QHO (taking into account the zero-point energy) was obtained by Einstein [29] in the form

$$
Z^{\mathrm{Q}}(c)=e^{-c}\left[1-e^{-2 c}\right]^{-1}=[2 \operatorname{sh} c]^{-1}=(1 / 2)[\psi(c)]^{1 / 2}
$$

having the asymptotes

$$
Z^{\mathrm{Q}}(c)= \begin{cases}Z^{\mathrm{C}}(c)\left[1-(1 / 6) c^{2}-O\left(c^{4}\right)\right], & (c \rightarrow 0), \\ e^{-c}+O\left(e^{-2 c}\right), & (c \rightarrow \infty) .\end{cases}
$$

Then according to definitions (2) and (3) the inner energy $E^{\mathrm{Q}}(c)$ and heat capacity $C_{v}^{\mathrm{Q}}(c)$ for $\mathrm{QHO}$ are given exactly by expressions $(25)$ and $(26)$ with $f(c)$ and $\varphi(c)$ defined by equation (39); using equations (40)-(42) their asymptotes may be easily compared with classic and semiclassic ones. Finally, according to equations (4), (25) and definition (37) of $\sigma_{0}$ the exact entropy of $\mathrm{QHO}$ is of the form

$$
\left(1 / k_{\mathrm{B}} \alpha\right) S^{\mathrm{Q}}(c)=\sigma_{0}+[f(c)-1]+(1 / 2) \ln \psi(c),
$$

which well satisfies the standard form of the Nernst theorem (namely, $S^{\mathrm{Q}}=0$ at $T=0)$.

\section{Bloch semiclassical approach}

A remarkably interesting and useful example of the value of the ET concept was actually given by Bloch [23], though Bloch himself did not explicitly employ this 
concept. Later on his result was reproduced in somewhat different way in [18] and [19], but, in our opinion, this remarkable result was not sufficiently commented from the physical point of view.

Bloch combined quantum mechanical (Born) and statistical mechanical (Gibbs) probabilistic descriptions on the general footing of von Neumann density operator approach. Thus Bloch obtained the exact quantum d.f.s separately in coordinate $q$ and momentum $p$ for the thermally equilibrium QHO.

Bloch's calculation is rather non-trivial and in all variants $[18,19,23]$ it includes the exact procedure of quantum summation. But it appears that Bloch's quantum d.f.s $P^{\mathrm{Q}}(q ; T)$ and $P^{\mathrm{Q}}(p ; T)$ may be easily obtained from their classical counterparts (32) and (33) by a simple use of the notion of Planck-Einstein ET. Indeed, making the substitution $T \rightarrow T^{*}(c)$ in the latter expressions we immediately obtain

$$
P^{\mathrm{Q}}(q ; T)=P^{\mathrm{C}}\left[q ; T^{*}(c)\right], \quad P^{\mathrm{Q}}(p ; T)=P^{\mathrm{C}}\left[p ; T^{*}(c)\right],
$$

where, naturally, $\alpha_{q, p}(T) \rightarrow \alpha_{q, p}\left(T^{*}\right)$ and the same is valid for $\sigma_{q, p}(T)$.

It is obvious, that in the high-T limit $\left(T^{*} \rightarrow T\right)$ we return again to the classical d.f.s (32) and (33) for CHO. But in the opposite low- $\mathrm{T}$ limit $\left(T \rightarrow 0, T^{*} \rightarrow T_{0}\right)$ we obtain

$$
\alpha_{p}\left(T_{0}\right)=1 / \hbar \omega, \quad \alpha_{q}\left(T_{0}\right)=\omega^{2} \alpha_{p}\left(T_{0}\right)=\omega / \hbar,
$$

and Bloch's d.f.s (46) go over to the well known Gaussian quantum probability densities for $q$ and $p$ in the ground state of QHO at $T=0$.

It is worth noting that due to the inequality (16) one has from the definition (35) that $\alpha_{q, p}\left(T^{*}\right) \leqslant \alpha_{q, p}(T)$. Then for the dispersions $\sigma_{q, p}^{2} \equiv 1 / 2 \alpha_{q, p}$ of d.f.'s (46) in $q$ and $p$ one always has

$$
\sigma_{q, p}^{2}\left(T^{*}\right) \geqslant \sigma_{q, p}^{2}(T), \quad \text { or } \quad\left[\sigma_{q, p}^{\mathrm{Q}}\right]^{2} \geqslant\left[\sigma_{q, p}^{\mathrm{C}}\right]^{2} .
$$

This means that the quantum d.f.s (46) are always wider than their classical counterparts (32) and (33), so the quantum fluctuations additionally to thermal ones "smear out" the statistical distributions in q and p. It is especially important for the limiting case $T=0, T^{*}=T_{0}$, when classical d.f.s degenerate into $\delta$-functions with zero dispersions, although quantum d.f.s remain Gaussian with finite dispersions:

$$
\sigma_{q, p}^{\mathrm{C}}(0)=0, \quad \sigma_{q}^{\mathrm{Q}}\left(T_{0}\right)=[\hbar / 2 \omega]^{1 / 2}, \quad \sigma_{p}^{\mathrm{Q}}\left(T_{0}\right)=[\hbar \omega / 2]^{1 / 2} .
$$

The difference between the classical (equal to zero) and pure quantum (finite and proportional to $\hbar$ ) values of dispersion at $T=0$ is, of course, completely due to the ground-state (i.e., vacuum) quantum fluctuations.

Some more relations may be also of use from the viewpoint of interrelations between the dynamic and thermodynamic descriptions. Forming the product of the dispersions $\sigma_{q}$ and $\sigma_{p}$, one obtains for the C-regime

$$
\left(\sigma_{q}^{\mathrm{C}} \sigma_{p}^{\mathrm{C}}\right)^{2}=\left(1 / 2 \hbar_{\mathrm{T}}^{\mathrm{C}}\right)^{2}, \quad \hbar_{\mathrm{T}}^{\mathrm{C}} \equiv \hbar / c,
$$

while for the $Q$-regime

$$
\left(\sigma_{q}^{\mathrm{C}} \sigma_{p}^{\mathrm{C}}\right)^{2}=\left(1 / 2 \hbar_{\mathrm{T}}^{\mathrm{Q}}\right)^{2}, \quad \hbar_{\mathrm{T}}^{\mathrm{Q}} \equiv \hbar \varphi(c) .
$$


In these expressions we have introduced the corresponding effective, or "thermal" Planck constants $\hbar_{\mathrm{T}}$ (strictly alike the introduction of "thermal" de Broglie wave length $\lambda_{\mathrm{T}}$ ) for both regimes considered. Note that $\hbar_{\mathrm{T}}^{\mathrm{C}}$ is rather formal quantity and may even not contain $\hbar$ itself - this is just the case for $\mathrm{HO}$, because due to definitions (50) and (8)

$$
\hbar_{\mathrm{T}}^{\mathrm{C}}=\hbar / c=\hbar\left(T / T_{0}\right)=T(2 / k \omega) .
$$

On the other hand, taking into account the asymptotic properties (40) of $\varphi(c)$ one obtains

$$
\hbar_{\mathrm{T}}^{\mathrm{Q}}= \begin{cases}\hbar_{\mathrm{T}}^{\mathrm{C}}\left[1+(1 / 3) c^{2}+O\left(c^{3}\right)\right], & (c \rightarrow 0), \\ \hbar\left[1+O\left(e^{-2 c}\right)\right], & (c \rightarrow \infty),\end{cases}
$$

so that $\hbar_{\mathrm{T}}^{\mathrm{Q}} \approx \hbar_{\mathrm{T}}^{\mathrm{C}}$ at high $T$, but $\hbar_{0}^{\mathrm{Q}}=\hbar>0$ in contrast to $\hbar_{0}^{\mathrm{C}}=0$ at $T=0$.

Note in conclusion that on the grounds of the result (51) thermally equilibrium states of $\mathrm{HO}$ described by the Bloch d.f.s (46) at $T=0$ go over into coherent states with the minimal property $\left(\sigma_{q}^{\mathrm{Q}} \sigma_{p}^{\mathrm{Q}}\right)^{2}=(\hbar / 2)^{2}$ (see papers [8] in this connection).

The remarkable simplicity in obtaining Bloch's results for quantum d.f.s from classical ones via the concept of ET makes it natural to raise the following question: will the calculation of p.f. ${ }^{17}$ using the same "recipe" of the substitution of ET in place of $T$,

$$
Z^{\mathrm{C}}(T) \rightarrow Z^{\mathrm{C}}\left[T^{*}(c)\right]
$$

also lead to the exact p.f. $Z^{\mathrm{Q}}(T)$ (or, at least, differ from it not very strong)?

Unfortunately, the answer is rather disappointing: the p.f. in rhs of equation (54), being of course different from $Z^{\mathrm{C}}(T)$ given by equation (36), does not coincide with $Z^{\mathrm{Q}}(T)$ given by equation (43). That is why p.f. in rhs of equation (54) should be considered only as an intermediate, or semiclassical one:

$$
Z^{\mathrm{C}}\left[T^{*}(c)\right] \equiv Z^{\mathrm{SC}}(c), \quad Z^{\mathrm{SC}}(c)=Z^{\mathrm{C}}(c) f(c)=(1 / 2) \varphi(c) ;
$$

nevertheless, we consider it as worth studying.

In particular, due to equation $(40) Z^{\mathrm{SC}}(c)$ possesses the following asymptotes (where we use the notation $Z_{\min }^{\mathrm{SC}}(\infty)=1 / 2$ ):

$$
Z^{\mathrm{SC}}(c)= \begin{cases}Z^{\mathrm{C}}(c)\left[1+(1 / 3) c^{2}+O\left(c^{4}\right)\right], & (c \rightarrow 0), \\ Z_{\min }^{\mathrm{SC}}(\infty)\left[1+O\left(e^{-2 c}\right)\right], & (c \rightarrow \infty) .\end{cases}
$$

On the other hand, it is easy to see that the exact p.f. $Z^{\mathrm{Q}}(c)$ for QHO from equation (43) may be written as

$$
Z^{\mathrm{Q}}(c)=Z^{\mathrm{SC}}(c) \Psi(c)
$$

where

$$
\Psi(c)=\left\{1-\left[Z_{\min }^{\mathrm{SC}}(\infty) / Z^{\mathrm{SC}}(c)\right]^{2}\right\}^{1 / 2}
$$

\footnotetext{
${ }^{17}$ In order to avoid any confusion note that Bloch [23] did not calculate the p.f. of QHO at all.
} 
Evidently, the factor (58) can by no means be "guessed" or anyhow deduced from equation (36) by means of ET (see also the footnote 10). The reason of such a discrepancy between equations (56) and (57) lies quite deep and concerns the very essence of the quantum description.

First of all let us note that the simple substitution meant in (54) implicitly suggests the fulfillment of the following rather strong (and in fact inadmissible in Q-regime) Ansatz:

The joint semiclassical d.f. in $q$ and $p$ should be of the same multiplicative form as it is in equation (29) for C-regime with the only change $T \rightarrow T^{*}(c)$. In other words, one should accept that

$$
P^{\mathrm{SC}}(q, p ; T)=P^{\mathrm{Q}}(q ; T) P^{\mathrm{Q}}(p ; T)=P^{\mathrm{C}}\left[q ; T^{*}(c)\right] P^{\mathrm{C}}\left[p ; T^{*}(c)\right],
$$

because only in this case the transition from (30) to (54) would be justified.

It should be noted that in C-regime the property (29) is quite acceptable due to two reasons: the first (general) is the very possibility of simultaneous definition (i.e., measuring) of $q$ and $p$, which is necessary in order to define the joint d.f.; the second (specific) is the separable structure of the Hamilton function (5), which causes the statistical independence of $q$ and $p$ fully expressed by equations (27)-(29).

However, in $Q$-regime these both reasons are violated and so the Ansatz (59) may be viewed at best as only giving some convenient "test" joint d.f. (and therefore also the p.f.) if - as is generally the case - one does not have the exact p.f. at one's disposal. In the considered here case of HO the exact p.f. in the form (43) fortunately exists, so the equations (55) and (59) are employed mainly for methodological purposes.

The physical reason of non-applicability of the Ansatz (59) as an exact one in $Q$-regime is called forth by the presence of quantum uncertainties $\sigma_{q}^{\mathrm{Q}}$ and $\sigma_{p}^{\mathrm{Q}}$, which according to equation (53) are of "saturated" Heisenberg form $\sigma_{q}^{\mathrm{Q}} \sigma_{p}^{\mathrm{Q}}=\hbar / 2$ at $T=0$. Due to this the quantities $q$ and $p$ are exactly defined only in their own representations, but they cannot be defined (and/or measured) simultaneously in any one of them. So the d.f.s (46) may well exist separately, but one should be very careful and thoroughly control the accuracy of results when using them simultaneously ${ }^{18}$ in the simple multiplicative form (60).

Summing up, the coordinates $q$ and momenta $p$ in $Q$-regime are somehow inherently correlated and so the Ansatz (60) is evidently not appropriate in the whole interval of $c$ (besides, maybe, only the "classical" high- $T$, or low- $c$ region). From equations (54)-(56) it is clear that an adequate (but of course quite formal) consideration of the quantum correlation between $q$ and $p$ may be achieved, if one introduces the factor (58) into equation (59) and defines the full quantum d.f. ${ }^{19}$ :

$$
P^{\mathrm{Q}}(q, p ; T)=P^{\mathrm{SC}}(q, p ; T)[\Psi(c)]^{-1} .
$$

\footnotetext{
${ }^{18}$ Note that the described in section 3 rather crude "cellular" method of considering the quantum indeterminacies is now sufficient and leads only to the correct form of classical $Z^{\mathrm{C}}$ (see (35)).

${ }^{19}$ Certainly, the representation (57) is only a formal trick because it demands to know already the exact quantum result (41), and in this case the whole idea of inventing some SC-approach of the type (52) becomes superfluous.
} 
Though not quite justified, we may adopt the p.f. (56) as a tool of desired intermediate or semiclassical description, because the results for its derivatives according to equations (2)-(4) may have (and does have) more sense than $Z^{\mathrm{SC}}(c)$ itself. It is important that having an exact expression $Z^{\mathrm{Q}}(c)$ we may easily check the accuracy of the "semiclassical" results on each step.

Due to asymptotic expansion (40) of $\varphi(c)$ one has according to (56) in the high- $T$ (low-c) region $Z^{\mathrm{SC}}(c) \gg Z_{\min }^{\mathrm{SC}}(\infty)$, so the factor (58) is very near to unity, and thus (up to corrections of the order $O\left(c^{2}\right)$ )

$$
Z^{\mathrm{C}}(c) \geqslant Z^{\mathrm{SC}}(c) \geqslant Z^{\mathrm{Q}}(c) \quad(c \rightarrow 0) .
$$

But in low- $T$ (high-c) region $Z^{\mathrm{SC}}(c) \rightarrow Z_{\mathrm{min}}^{\mathrm{SC}}(\infty)=1 / 2$ and the factor (58) plays a decisive role forcing $Z^{\mathrm{Q}}(c)$ (in contrast to $Z^{\mathrm{SC}}(c)$ ) to go to zero exponentially fast. Paradoxically enough, but in the low- $T$ region $Z^{\mathrm{C}}$ has the same limiting value (equal to zero) as $Z^{\mathrm{Q}}$ has, but with wrong asymptotes, whereas $Z^{\mathrm{SC}}$, on the contrary, has a wrong (finite $=1 / 2$ ) limiting value, but correct asymptotic behavior.

The thermodynamic functions (2)-(4) in our "quasi-Bloch's" SC-approach are of the form

$$
\begin{gathered}
E^{\mathrm{SC}}(c)=E^{0}[\psi(c) / \varphi(c)], \quad C_{\mathrm{V}}^{\mathrm{SC}}(c)=C_{\mathrm{V}}^{\mathrm{Q}}(c)\left[\varphi^{2}(c)+1\right], \\
\left(1 / k_{\mathrm{B}}\right) S^{\mathrm{SC}}(c)=\sigma_{0}+[c \psi(c) / \varphi(c)-1]+\ln \varphi(c) ;
\end{gathered}
$$

their asymptotes may be easily obtained by means of expansions (40)-(42) and it may be seen that quantities (62) behave much better - i.e., resemble more (though not fully) the exact quantities (25), (26) and (45) than do the classical ones (23) and (37) in the most important high-c region (in particular, $C_{\mathrm{V}}^{\mathrm{SC}} \rightarrow 0$ and $S^{\mathrm{SC}} \rightarrow$ $\sigma_{0}-1=-\ln 2$ as $\left.T \rightarrow 0\right)$.

\section{Wigner semiclassical approach}

Wigner [22] originally introduced (with the assistance of Szilard) the so-called quasiprobability, or Wigner function $W(q, p)$ in phase space thus assuming from the very beginning the possibility of simultaneously determining $p$ and $q$; after suitable integration this function gives in principle the exact d.f.s $P^{\mathrm{Q}}(q ; T)$ and $P^{\mathrm{Q}}(p ; T)$.

But by means of $W(q, p)$ Wigner elaborated only the expansions ${ }^{20}$ (formal) of the statistical d.f.s and p.f. in powers of $\hbar$. In contrast to Bloch, Wigner was not restricted by the case of $\mathrm{HO}$, but considered the general case (5) with an arbitrary potential $U(q)$, which should be only an analytic function of $q$.

Wigner's approach proves to be in fact not only a "small-h" one, but (which is perhaps more important) rather a high- $T$ (or low-c) expansion. This is not surprising,

\footnotetext{
${ }^{20}$ In search of maximal physical transparence we present here just the original version of Wigner's approach [22] in the convenient form given to it by Landau and Lifshitz [18] (for more refined form see $[24,25]$. Unfortunately, Wigner's and Bloch's results were not compared in [18] with each other nor with the exact Planck-Einstein result even in the simplest case of quantum harmonic oscillator (QHO).
} 
since according to definition (8) $c \sim E_{0}$, where always $E_{0} \rightarrow 0$ at $\hbar \rightarrow 0$ (e.g., $E_{0}=O(\hbar)$ for $\mathrm{HO}, E_{0}=O\left(\hbar^{2}\right)$ for free particle in a box etc.). By this consideration it becomes clear that the opposite region of low- $T$ (or high-c) cannot in principle be described by such an expansion (see, however, extensive general discussion about more general Wigner-like expansions in $[18,19])$.

Nevertheless, the original Wigner's approach is rather simple and highly interesting and leads to the results cited below. The d.f.s $P_{\mathrm{W}}(q ; T)$ and $P_{\mathrm{W}}(p ; T)$ in coordinates and momenta are of the following form:

$$
\begin{aligned}
P_{\mathrm{W}}(q ; T) & \sim \exp \left\{-\left(1 / k_{\mathrm{B}} T\right) U_{\mathrm{W}}(q ; T)\right\}, \\
P_{\mathrm{W}}(p ; T) & \sim \exp \left\{-\left(p^{2} / 2 k_{\mathrm{B}} T\right)\left[1-\left[\hbar^{2} / 12\left(k_{\mathrm{B}} T\right)^{3}\right]\left\langle(\partial U(q) / \partial q)^{2}\right\rangle C\right]\right\},
\end{aligned}
$$

(for simplicity we present them only for one particle in the external potential $U(q)$ and only up to terms $O\left(\hbar^{2}\right)$ omitting $\left.O\left(h^{4}\right)\right)$.

Here classical Gibbs averaging with account of equation (6) means as usual

$$
\langle\cdots\rangle_{C}=\left[Z^{\mathrm{C}}(T)\right]^{-1} \int \mathrm{d} q \mathrm{~d} p /(2 \pi \hbar)^{s} \exp \left[-H(q, p) / k_{\mathrm{B}} T\right]
$$

finally, the Wigner's effective potential $U_{\mathrm{W}}(q ; T)$ has the form

$$
U_{\mathrm{W}}(q ; T)=U(q)+\left[\hbar^{2} / 12\left(k_{\mathrm{B}} T\right)\right]\left[\partial^{2} U(q) / \partial q^{2}-\left(1 / 2 k_{\mathrm{B}} T\right)(\partial U(q) / \partial q)^{2}\right] .
$$

Note that in general both d.f.s (63) and (64) depend upon the potential derivatives, which is quite alien to the classical statistical mechanics. But in quantum statistical mechanics it reflects the growth of kinetic energy (described by the operator $\left(\sim h^{2}\left[\partial^{2} / \partial q^{2}\right]\right)$ in $q$-representation $)$ in those space regions, where the potential energy $U(q)$ changes sharply enough ${ }^{21}$.

With the d.f.s (63) and (64) the joint semiclassical d.f. in Wigner's approach quite similar to Bloch's one (59) - may be written as their product:

$$
P_{\mathrm{W}}^{\mathrm{SC}}(q, p ; T)=P_{\mathrm{W}}(q ; T) P_{\mathrm{W}}(p ; T) .
$$

Then the Wigner's p.f. has the form

$$
Z_{\mathrm{W}}^{\mathrm{SC}}(T)=Z^{\mathrm{C}}(T)\left[1+\zeta(T)+O\left(\hbar^{4}\right)\right],
$$

where in obtaining the correction term

$$
\zeta(T)=\left(1 / k_{\mathrm{B}} T\right)\left\langle U_{\mathrm{W}}(q ; T)-U(q)\right\rangle C=-\left(\hbar^{2} / 24\right)\left(1 / k_{\mathrm{B}} T\right)\left\langle\partial^{2} U(q) / \partial q^{2}\right\rangle C
$$

\footnotetext{
${ }^{21}$ Note, that it is possible to write the exact quantum Bloch coordinate d.f. (46) in terms of effective potential in the form like (59) with the substitution $U_{\mathrm{W}}(q, T) \rightarrow U_{\mathrm{B}}(q, T)=U(q)[f(c)]^{-1}$ (where $f(c)$ is defined by equation (38)); clearly, no gradients of $U(q)$ enter explicitly $U_{\mathrm{B}}(q, T)$. But it appears more appropriate to rewrite, on the contrary, equation (59) in terms of Wigner's ET (defined below in equation (74)) as it also takes place in Wigner's momenta d.f. (64) written in the form (69).
} 
we used the following integration by parts from equation (65) relation

$$
\left\langle(\partial U(q) / \partial q)^{2}\right\rangle C=k_{\mathrm{B}} T\left\langle\partial^{2} U(q) / \partial q^{2}\right\rangle C .
$$

Thus the sign of the correction term (69) depends on the form of $U(q)$.

Let us now turn to the special case of $\mathrm{HO}$, where

$$
U(q)=(1 / 2) \omega^{2} q^{2}, \quad \partial U(q) / \partial q=\omega^{2} q, \quad \partial^{2} U(q) / \partial q^{2}=\omega^{2}=\text { const. }
$$

In this case the effective potential (66) (omitting the term independent of $q$, which effects only the beginning of the energy scale and so the normalization constant in p.f.'s) takes the form:

$$
U_{\mathrm{W}}(q ; T)=U(q)\left[1-h^{2} \omega^{2} / 12\left(k_{\mathrm{B}} T\right)^{2}\right]=U(q)\left[1-(1 / 3) c^{2}\right] .
$$

Then the d.f. (63) may be written in an equivalent form:

$$
P_{\mathrm{W}}(q ; T)=P^{\mathrm{C}}\left[q ; T_{\mathrm{W}}^{*}(c)\right],
$$

where instead of Wigner's effective potential (72) one may now introduce Wigner's effective temperature

$$
T_{\mathrm{W}}^{*}(c) \equiv T\left[1+(1 / 3) c^{2}\right]
$$

Further, let us note that

$$
\left\langle(\partial U(q) / \partial q)^{2}\right\rangle_{C}=\omega^{4}\left\langle q^{2}\right\rangle_{C}, \quad\langle U(q)\rangle_{C}=(1 / 2) \omega^{2}\left\langle q^{2}\right\rangle_{C}=(1 / 2) k_{\mathrm{B}} T,
$$

where in the latter equality we have used the classical theorem (22) of energy equipartition (for $\mathrm{HO}$, the contribution of potential and kinetic energies are equal).

So we have

$$
\left\langle q^{2}\right\rangle_{C}=k_{\mathrm{B}} T / \omega^{2}, \quad\left\langle(\partial U(q) / \partial q)^{2}\right\rangle_{C}=k_{\mathrm{B}} T \omega^{2},
$$

and finally the d.f. (64) obtains the form quite analogous to equation (73):

$$
P_{\mathrm{W}}(p ; T)=P^{\mathrm{C}}\left[p ; T_{\mathrm{W}}^{*}(c)\right]
$$

with the same Wigner's ET (74).

Using definition (16) and upper line of expansion (41) one can immediately see that the Wigner's (approximate) ET is nothing else but high- $\mathrm{T}$ expansion of the Planck-Einstein (exact) ET (39) (also coinciding with ET (46) used by Bloch).

It is further obvious that on the grounds of the last expression in (75) the correction term (69) for the p.f. may be written as

$$
\zeta(T)=-(1 / 6) c^{2}, \quad\left(c=\hbar \omega / 2 k_{\mathrm{B}} T\right) .
$$

Thus Wigner's p.f. (68) for QHO coincides with the first high- $T$ (low-c) correction term of the exact $Z^{\mathrm{Q}}$ (see the upper line of expansion (44)), but not Bloch's semiclassical $Z_{\mathrm{B}}^{\mathrm{SC}}$. Indeed, with equation (77) the expression (68) differs from the upper line (high- $T$ ) of expansion (56) in sign and numerical coefficient 2. 
Let us remark in conclusion that Wigner's approach based originally on "small $h$ " perturbation theory is, in general, of great value in quasiclassical dynamic problems (see, e.g., $[18,19]$ ), but has much more restricted efficiency in the statistical mechanical, or thermodynamic ones. The origin of this seems to lie in an essentially different nature of dimensionless parameters, which one may consider as small in these two cases.

More specific, in dynamic case the role of such a parameter is played by the ratio of actions $\hbar / S$, where $S$ is the characteristic value of the action in the considered problem. With $\hbar$ being the minimal value, or "quantum" of action this parameter is always of the order of unity or less; for the objects in almost classical regime, $S$ is large, so $\hbar / S \ll 1$, which formally may be used as the limit of $\hbar \rightarrow 0$.

However, in statistical mechanical problems, the action ceases to be a relevant physical quantity, so $\hbar$ is no more a single fundamental constant. As follows from the idea of effective temperature (see especially sections 4 and 5), in these problems the true physical dimensionless parameter is the ratio of energies $c=E_{0} / E_{\text {th }}$ (introduced in equation (8)), where $E_{0}$ is of the order $\hbar$ and characterizes the object itself.

But the quantity $E_{\mathrm{th}}=k_{\mathrm{B}} T$ depends upon the object's macroscopic surroundings, or thermostat, through its temperature $T$ and plays the role of some average thermal energy per one degree of thermostat's freedom; note, that by means of $E_{\mathrm{th}}$ another fundamental (Boltzmann) constant $k$ comes into play.

Contrary to the dynamic action " $S$ " the thermal energy $E_{\text {th }}$ possesses no natural non-zero limitations from below, whereas $E_{0}$ sometimes may be very small (but nonzero). The point is that $E_{0}$, though being proportional to $\hbar$, is itself not a universal constant, thus allowing the values $E_{\text {th }} \leqslant E_{0}$, or $T \leqslant T_{0}$, the classical regime for the quantum object is possible when $E_{\mathrm{th}} \gg E_{0}, c \ll 1$, and so this regime may be reproduced formally by means of the limit $\hbar>0$.

However, in the opposite case, when $E_{\text {th }} \ll E_{0}$ (so that $\mathrm{T}$ is low enough) the parameter $c \gg 1$ and thus no kind of expansion in $\hbar$ is in principle allowed and applicable. Let us emphasize once more that the failure of Wigner's original semiclassical approach in the low- $T$ region of quantum regime is due to the presence of the outer (relative to the described object) parameter $T$, which may be made arbitrary small (though staying non-negative).

In this region the exact $(\mathrm{Q})$ results for thermodynamic functions differ from the classical $(\mathrm{C})$ ones by the correction terms of the order $O\left(e^{-c}\right)$, which are nonanalytical in $c$ at $c \rightarrow \infty$ (low-T limit). Clearly, in this limit the Planck constant $\hbar$ can in no way (even formally) be considered as a "small" one.

In conclusion, it is instructive to note the following. As is well known [22,24,25], the Wigner's d.f.s for the dynamic quantum systems (e.g., for QHO) possess another hard disadvantage: they are negative for some excited states of QHO, whereas for the ground-state the Wigner's d.f.s are strongly positive and coincide with Bloch ones.

However, for the thermodynamic quantum object (i.e, QHO in thermal contact with thermostat) the Wigner's d.f.s (63) and (64) are always positive. This can be considered as if the thermostat induces the additional incoherence and so makes 
Wigner's (as well as Bloch's, see equation (48)) d.f.s more smooth and wide spread at the same time.

\section{Conclusions}

In this paper we investigated the applicability of the concept of universal effective temperature (ET) anticipated by Kirchhoff and Wien and first deduced and applied by Planck and Einstein to the thermal radiation. The latter authors studied the exact (in the sense of quantum statistical mechanics) description of the set of harmonic oscillator being in thermal equilibrium with the thermostat at temperature $T$. Much earlier this problem was in fact formulated by Maxwell and Boltzmann on the grounds of classical statistical mechanics description; their results proved to be approximate and valid only in the high- $T$ region.

The aim of our paper was to establish some intermediate, or semiclassical description following the seminal ideas of Bloch and Wigner. Namely, we considered the thermodynamic consequences of substituting the Planck-Einstein ET instead of $T$ into Maxwell-Boltzmann formulas, which is equivalent to Bloch-Wigner approach.

We have shown that semiclassical thermodynamic quantities, i.e., inner energy, heat capacity and entropy obtained in this way, agree qualitatively with their exact quantum counterparts much better than the classical ones. So one may consider the concept of effective temperature as the effective tool for approximate "quantization" of thermodynamic functions (i.e., partially considering the quantum fluctuations additionally to thermal ones) obtained on the grounds of Gibbs classical statistical mechanics.

Note, that the more general question of failure of the Gibbs approach itself at very low $T$ due to very large temperature fluctuations was raised recently [16]; evidently, this may demand to go over from Gibbs to Einstein approach (or, in other terms, from SC- to NC- theory on the T-level, see section 2).

Nevertheless, we hope that the method suggested by us will appear useful not only for the well-known example of harmonic oscillator (which fortunately possesses the exact solution and permits to test the accuracy of any approximation), but also for other less simple objects.

In particular it may well be applied to compound systems, consisting of some dynamically non-interacting subsystems of quantum origin, each of them having its own ground-state energy. As a good example of this kind may serve the microscopic particle with inner degrees of freedom (besides the translational ones) - e.g., the diatomic molecule with rotations, vibrational and electronic degrees of freedom.

In general we assume that the method of ET is applicable to arbitrary quantum systems though it is delicate enough and in every case deserves a careful analysis. The applicability of various approximations should be verified and their relative errors accurately evaluated which is a far not trivial task in the absence of the exact solution. But, as we hope, an important specific case of the harmonic oscillator studied here will give us some valuable hints due to universal applicability of PlanckEinstein ET at least, it is valid for the large class of objects, possessing no upper 
bound for their energy and so not permitting the negative values of $T$ (such as objects ore, e.g., the spin systems). Indeed, it appears even in the quantum generalizations of the Langevin equation and the fluctuation-dissipation theorem (see, e.g., $[3,18]$ ).

From the practical point of view it is very significant at what stage ET should be inserted into classical thermodynamic functions in order to obtain their correct asymptotic behavior in both limits of high $\left(T / T_{0} \rightarrow \infty\right)$ as well as low $\left(T / T_{0} \rightarrow 0\right)$ thermostat temperatures. For example, if the substitution $T \rightarrow T^{*}$ is performed in the partition function (as is made in the paper), in the high- $T$ limit all three approaches - classical, semiclassical and quantum - give approximately the same results, differing only by small correction terms of the order $\left(T / T_{0}\right)^{2}$.

On the other hand, at low- $\mathrm{T}$ limit quantum as well as - which is especially important - semiclassical approaches give results, that are exponentially small in $\left(T_{0} / T\right)$ and thus radically different from the classical one, for which $T_{0} \rightarrow 0$. In this way the important physical requirement - namely, the fulfillment of Nernst theorem - is properly guaranteed. One should note that the same substitution $T \rightarrow T^{*}$ in the classical expressions for inner energy leads (probably accidentally) to the exact result, whereas such a procedure for the specific heat capacity is not possible at all because $C_{\mathrm{V}}^{\mathrm{C}}$ does not contain $T$.

In conclusion let us comment on the choice between the Wigner and Bloch forms of the semiclassical (in the sense of considering not thermal but only quantum fluctuations). As was shown in [24], the modified Wigner's approach is eventually equivalent to the Bloch's one; this is not surprising, because the presentation in [24] is based on the phase space interpretation of Bloch equation for von Neumann density operator.

In our opinion, the Bloch's approach is something more practical and convenient. But its difficulty consists in the necessity of knowing not only the form of the object's potential energy, but also of the full Hamiltonian eigenvalues needed for the calculation of Gibbs factors entering the quantum partition function.

In any case, the original Wigner's approach (which is available in valid textbooks) is applicable only in the high-T limit, and this fact is not always sufficiently stressed. On the other hand, the use of Wigner's approach for "warm" quantum objects is more justified because the effect of the thermostat eliminates one of the greatest difficulties in this approach. Namely, the thermal fluctuations induced by thermostat lead to additional incoherence and thus "smooth out" and "rectify" Wigner functions restoring their positivity.

In the subsequent papers we shall try to apply the semiclassical approach in the form of effective temperature to some more examples of "warm" quantum objects. Moreover, we intend to study the generalized case when the thermostat itself is partially quantized by means of its own effective temperature.

\section{Acknowledgement}

The authors devote this paper in honour of Prof. Dr. Igor R. Yukhnovsky on the occasion of his 80th birthday. We evaluate very much the fruitful efforts, which 
Prof. Yukhnovsky has spent to continue and elaborate the ideas of N.N.Bogoliubov's school in the field of modern statistical mechanics. The present authors are also indebted to the late Prof. Dr. Yu.L.Klimontovich with whom we have had interesting discussions on the theory of open quantum systems.

\section{References}

1. Rudoy Yu.G., Sukhanov A.D., Uspekhi Fiz. Nauk, 2000, 170, 1265 (in Russian). Engl. transl.: Phys. Uspekhi, 2000, 43(12), 1169.

2. Giulini D., Joos E., Zeh H.D., Decoherence and the Appearance of Classical World in Quantum Theory. Springer, Heidelberg, 1996.

3. Klimontovich Yu.L., The Statistical Theory of Open Quantum Systems, vol. 1. Yanus, Moscow, 1995 (in Russian). Engl. transl.: Kluwer Academic Publishers, Dordrecht, 1995.

4. Kadomtsev B.B., Dynamics and Information. UFN Publishing House, Moscow, 1997 (in Russian).

5. Sukhanov A.D., Golubeva O.N., Physical Education in High School, 2001, 7, 16 (in Russian).

6. Einstein A., Ann. Phys. Leipzig, 1903, 11, 170.

7. Lavenda B., Int. J. Theor. Phys., 1987, 26, 1069; Schlogl F., J. Phys. Chem. Sol., 1988, 49, 679; Uffink J., van Lith J., Found. Phys., 1988, 28, 323.

8. Sukhanov A.D., Theor. and Math. Phys., 2000, 125, 1489; Physics of Particles and Nuclei, 2001, 32, 619.

9. Szilard L., Z. Phys., 1925, 32, 753.

10. Mandelbrot B., Ann. Math. Stat., 1962, 33, 1021; J. Math. Phys., 1964, 5, 164.

11. Tisza L., Quay P.M., Ann. Phys., 1963, 25, 48.

12. Petrov N., Brankov Y., The Modern Problems of Thermodynamics. Mir, Moscow, 1986 (in Russian).

13. Gibbs J.W., Elementary Principles in Statistical Mechanics. Scribner, NY, 1902.

14. The Conceptual Foundations of Quantum Field Theory, ed. T.Y.Cao. Cambridge University Press, Cambridge, 1999.

15. Penrose R., The Emperor's New Mind. Cambridge University Press, Cambridge, 1989.

16. Wu J., Widom A., Phys. Rev. E, 1998, 57, 5178; Nieuwanheuizen Th.M., Allahverdyan A.E., Phys. Rev. E, 2002, 66, 036102.

17. Einstein A., Ann. Phys. Leipzig, 1910, 33, 1275.

18. Landau L.D., Lifshitz E.M., Statistical Physics. GTTI, Moscow, 1939 (in Russian). English transl.: Addison-Wesley, Reading, Mass., 1958, Ch. III.

19. Leontovich M.A., Statistical Physics. GTTI, Moscow, 1944, 2nd ed.: Nauka, Moscow, 1983 (in Russian), Ch. III.

20. Zubarev D.N., Nonequilibrium Statistical Thermodynamics. Nauka, Moscow, 1971 (in Russian). German transl.: D.N.Zubarev, Statistische Thermodynamic der Nichtgleichgewicht. Akademie Verlag, Berlin, 1976.

21. Huang K., Statistical Mechanics. John Wiley, New York- London, 1963, Ch. 10.

22. Wigner E.P., Phys. Rev., 1932, 40, 749.

23. Bloch F., Z. Phys., 1932, 74, 295.

24. Hillery M., O'Connell R.F., Scully M.O., Wigner E.P., Phys. Rep., 1984, 106, 121. 
25. O'Connell R.F., Found. Phys., 1983, 13, 83; Lee H.W., Scully M.O., Found. Phys., 1983, 13, 61.

26. Sukhanov A.D., On the global interrelation between quantum dynamics and thermodynamics. - In: Proc. of the XI Int. Conf. "Problems of Quantum Field Theory", 1998. Dubna, JINR, 232, 1999.

27. Planck M., Verhandl. Deutsch. Phys. Ges., 1900, 2, 202, 237; Ann. Phys., 1901, 4, 553.

28. Planck M., Verhandl. Deutsch. Phys. Ges., 1911, 13, 138.

29. Einstein A., Verhandl. Deutsch. Phys. Ges., 1914, 16, 820.

30. Wien W., Sitzungsber. Deutsch. Akad. Wiss. Berlin, (vom 9 Februar 1893, S. 55); Ann. Phys., 1901, 4, 553.

31. Kirchhoff G., Ann. Phys., 1860, 19, 275.

\title{
До єдиної некласичної фізики: врахування квантових флуктуацій в рівноважній термодинаміці через ефективну температуру
}

\author{
Ю.Г.Рудой, А.Д.Суханов
}

Університет дружби народів , 117198, вул. Міклухи-Маклая, 6, Москва, Росія

Отримано 13 травня 2005 р.

Концепція ефективної температури (ЕT) $T^{*}\left(T_{0}, T\right)$ використовується для того, щоб наближено "квантувати" термодинамічні функції динамічного об'єкту, який є в термічній рівновазі з термостатом при постійній температурі $\boldsymbol{T}\left(\boldsymbol{T}_{\mathbf{0}}=\boldsymbol{E}_{\mathbf{0}} / \boldsymbol{k}_{\mathrm{B}}\right.$, де $\boldsymbol{E}_{0}-$ енергія основного стану, $\boldsymbol{k}_{\mathrm{B}}$ - постійна Больцмана, $-\epsilon$ характеристичною "квантовою" температурою самої системи). Базуючись на цьому, здійснено екстенсивне порівняння дослідження “стандартної моделі" статистичної механіки - одновимірного гармонійного осцилятора (ГО). Розглянуто три добре відомі підходи та добре вивчено іх термодинамічні наслідки, а саме: точний квантовий або некласичний підхід ПланкаЕйнштейна, проміжний або напівкласичний підхід Блоха-Вігнера i, нарешті, чисто класичний або підхід Максвелла-Больцмана.

Ключові слова: рівноважна термодинаміка, ефективна температура, квантові флуктуації

PACS: 05.70.-a, 05.30.-d, 05.40.-a 
